\title{
The spacetime in the neighborhood of a general isolated black hole
}

\author{
Badri Krishnan ${ }^{1,2}$ \\ ${ }^{1}$ Max Planck Institute for Gravitational Physics, Albert Einstein Institute, Am \\ Mühlenberg 1, D-14476 Potsdam, Germany \\ ${ }^{2}$ Max Planck Institute for Gravitational Physics, Albert Einstein Institute, \\ Callinstrasse 38, D-30167 Hannover, Germany \\ E-mail: badri.krishnan@aei.mpg.de
}

\begin{abstract}
We construct the spacetime in the vicinity of a general isolated, rotating, charged black hole. The black hole is modeled as a weakly isolated horizon, and we use the characteristic initial value formulation of the Einstein equations with the horizon as an inner boundary. The spacetime metric and other geometric fields are expanded in a power series in a radial coordinate away from the horizon by solving the characteristic field equations in the Newman-Penrose formalism. This is the first in a series of papers which investigate the near horizon geometry and its physical applications using the isolated horizon framework.
\end{abstract}

\section{Introduction}

The intrinsic geometry of a classical black hole horizon in equilibrium is well understood. The most general treatment is provided by the framework of isolated horizons which allows for the possibility of a black hole with arbitrary (but time independent) intrinsic geometry in an otherwise dynamical spacetime [1, 2, 3, 4, 5, 6, 7]. Isolated horizons have been applied in various physical circumstances. Some illustrative examples are black hole thermodynamics, black hole entropy calculations in quantum gravity, numerical relativity, and hairy black holes. More general situations when the black grows due to infalling matter and/or radiation have also been studied; see e.g. [8, 9]. See [10, 11, 12, 13] for reviews and references.

In order to use isolated horizons in other astrophysical scenarios, it is important to calculate the metric in some neighborhood of the black hole. Thus it is interesting to solve the Einstein equations in the neighborhood of a black hole horizon. Such calculations have been carried out in the astropysically interesting context of a Schwarzschild or Kerr black hole which is tidally deformed due to its environment [14, 15, 16, 17, 18]. Our approach here is closest to the work of Poisson and collaborators [15, 16] who study the tidal deformation of a non-rotating black hole using coordinates based on the past light cones originating from the horizon. These coordinates were originally described in [4] and are similar to the Bondi coordinates near null infinity 
[19]. The present paper goes towards generalizing [15, 16] to include rotation, electric charge, and higher multipoles.

We use the characteristic initial value formulation of Einstein's equations where free data is specified on a set of intersecting null hyper-surfaces [20, 21, 22, 23]. Consider $N$ dependent variables $\psi_{I}(I=1, \ldots, N)$ on a spacetime manifold with coodinates $x^{a}$. We shall be concerned with hyperbolic first-order quasilinear equations of the form

$$
\sum_{J=1}^{N} A_{I J}^{a}(x, \psi) \partial_{a} \psi_{J}+F_{I}(x, \psi)=0 .
$$

In the standard Cauchy problem, one specifies the $\psi_{I}$ at some initial time. A solution is then guaranteed to be unique and to exist at least locally in time. The characteristic formulation considers a pair of null surfaces $\mathcal{N}_{0}$ and $\mathcal{N}_{1}$ whose intersection is a codimension-2 spacelike surface $S$. It turns out to be possible to specify appropriate data on the null surfaces and on $S$ such that the above system of equations is well posed and has a unique solution, at least locally near $S$.

In our case, the appropriate free data is specified on the horizon and on an outgoing past light cone originating from a cross section of the horizon. Such a construction in the context of isolated horizons was first studied by Lewandowski [24] who characterized the general solution of Einstein equations admitting an isolated horizon. The general scenario is sketched in Figure1. We consider a portion of the horizon $\Delta$ which is isolated, in the sense that no matter and/or radiation is falling into this portion of the horizon. For a cross-section $S$, the past-outgoing light cone is denoted by $\mathcal{N}$. The null generators of $\Delta$ and $\mathcal{N}$ are parameterized by $v$ and $r$ respectively; $x^{i}$ are coordinates on $S$. This leads to a coordinate system $\left(v, r, x^{i}\right)$ which is valid till the null geodesics on $\mathcal{N}$ start to cross. The field equations are solved in a power series in $r$ away from the horizon. This construction will be spelled out more precisely in the course of this paper.

Unlike in [15, 16] we use the Newman-Penrose formalism [25] which, as we shall see, is well suited to this problem because of the central role played by null surfaces. Furthermore, including rotation and electric charge do not make the equations much more complicated in the Newman-Penrose framework. The results of this paper cannot yet be directly compared with [15, 16]. This would require us to start with, say a Kerr black hole, perturb it by considering a non-vanishing background curvature, and to expand the perturbation in powers of $r$. Perturbation theory turns out to be straightforward in this general set-up, at least in principle. This will be studied in a forthcoming paper. We make no attempt to study the issue of global existence of solutions, and our solutions are valid only in a neighborhood of $\Delta$. There is no guarantee, for example, that our solutions could be extended out to an asymptotically flat region. Nevertheless, we expect that our construction does include most solutions of possible astrophysical relevance. In fact, it can be shown numerically [26] that for a Kerr black hole, this coordinate system extends all way out to past null infinity. It is thus reasonable to expect that for perturbations of Kerr, the coordinate system extends sufficiently far away from the horizon. 


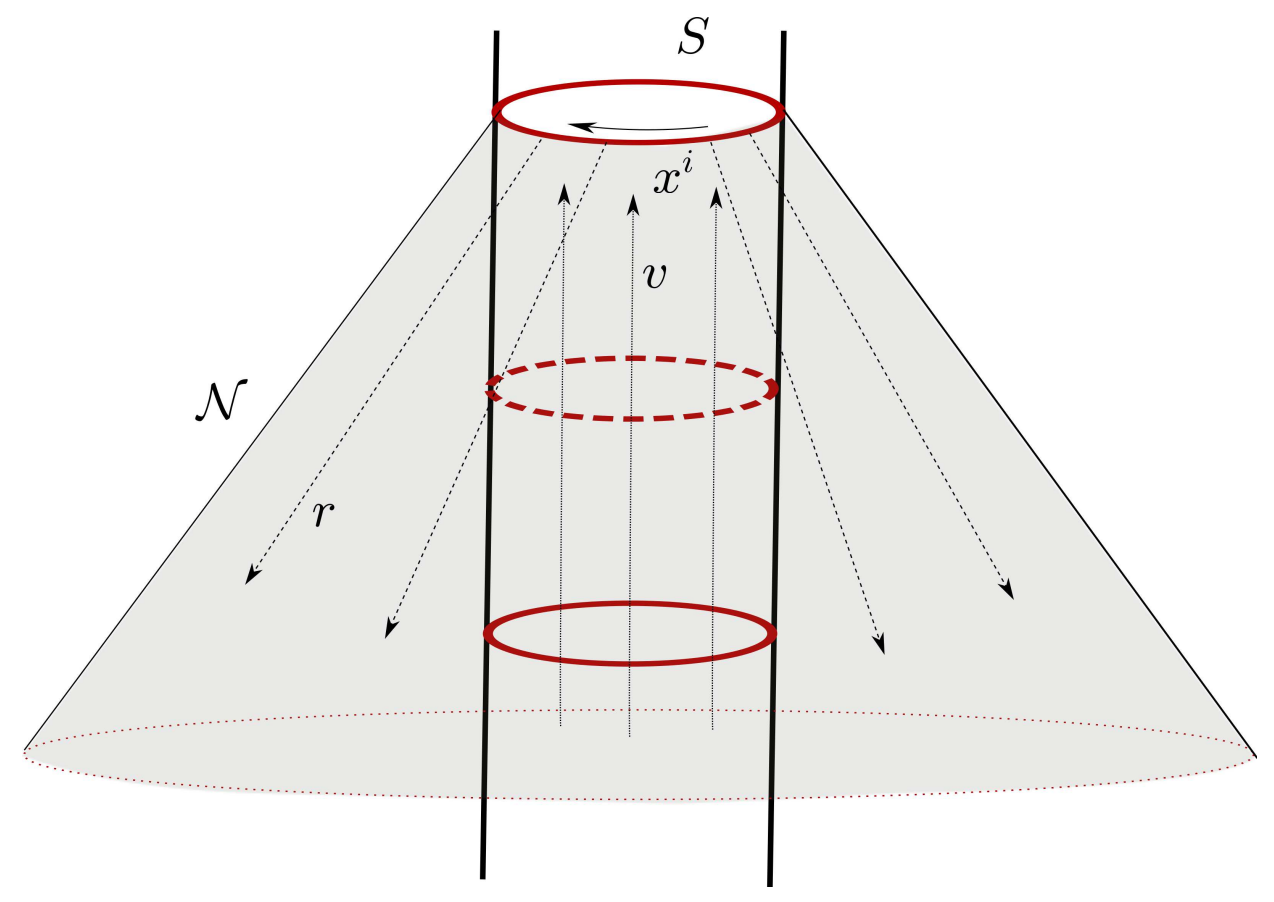

Figure 1. The near horizon coordinate system. The null generators of the horizon are parameterized by $v$, surfaces of constant $v$ are spheres. On any such sphere choose coordinates $x^{i}$. The past directed outgoing light cone originating from $S$ is $\mathcal{N}$ and $r$ an affine parameter along the null generators of $\mathcal{N}$.

The plan for this paper is as follows. Section 2 reviews the Newman-Penrose formalism and summarizes the definitions and some basic properties of non-expanding and weakly isolated horizons. This will form the basis of the inner boundary conditions that will be imposed later. Section 3 sets up the near horizon coordinate system and gauge conditions. We start by ignoring matter fields which will be included later in Section 8. Section 4 summarizes the Einstein field equations and the Bianchi identities for vacuum spacetimes in the Newman-Penrose formalism, and Sec. 6] explicitly solves the field equations in powers of the radial coordinate $r$. Section 8 considers EinsteinMaxwell theory by incorporating the source free Maxwell equations. This section points out the specific aspects of the previous calculations which need to be modified due to the presence of an electromagnetic field. Finally Sec. 9 concludes with a summary and suggestions for further investigations.

For simplicity, all manifolds and geometric fields shall be assumed to be smooth. We work in units where $G=c=1$. We use the abstract index notation where lower case Latin letters $a, b, \ldots$ are 4 -dimensional spacetime indices, and $i, j, \ldots$ denote the 2-dimensional angular directions. We take the spacetime metric $g_{a b}$ to have a signature $(-+++)$. The Riemann tensor $R_{a b c d}$ is defined by $\left(\nabla_{a} \nabla_{b}-\nabla_{b} \nabla_{a}\right) X^{c}=-R_{a b d}^{c} X^{d}$ where $\nabla_{a}$ is the derivative operator compatible with $g_{a b}$ and $X^{a}$ is an arbitrary smooth vector field. 


\section{Basic notions}

In this section, for completeness and to set up notation, we briefly review the NewmanPenrose formalism and the basic definitions and properties of non-expanding and weakly isolated horizons.

\subsection{The Newman-Penrose formalism}

The Newman-Penrose formalism [25, 27] is a tetrad formalism where the tetrad elements are null vectors, which makes it especially well suited for studying null surfaces. See [28, 29, 23] for pedagogical treatments (note that these references take the spacetime metric to have a signature of $(+---)$ which is different from ours). Start with a null tetrad $(\ell, n, m, \bar{m})$ wher $\ell$ and $n$ are real null vectors, and $m$ is a complex null vector and $\bar{m}$ its complex conjugate. The tetrad is such that $\ell \cdot n=-1, m \cdot \bar{m}=1$, with all other inner products vanishing. The spacetime metric is thus given by

$$
g_{a b}=-\ell_{a} n_{b}-n_{a} \ell_{b}+m_{a} \bar{m}_{b}+\bar{m}_{a} m_{b} .
$$

Directional derivatives along the basis vectors are denoted as

$$
D:=\ell^{a} \nabla_{a}, \quad \Delta:=n^{a} \nabla_{a}, \quad \delta:=m^{a} \nabla_{a}, \quad \bar{\delta}:=\bar{m}^{a} \nabla_{a} .
$$

(The symbol $\Delta$ is used for both the directional derivative along $n^{a}$ and for the isolated horizon). The components of the connection are encoded in 12 complex scalars, the spin coefficients, defined via the directional derivatives of the tetrad vectors:

$$
\begin{cases}D \ell & =(\epsilon+\bar{\epsilon}) \ell-\bar{\kappa} m-\kappa \bar{m}, \\ D n & =-(\epsilon+\bar{\epsilon}) n+\pi m+\bar{\pi} m, \\ D m & =\bar{\pi} \ell-\kappa n+(\epsilon-\bar{\epsilon}) m, \\ \Delta \ell & =(\gamma+\bar{\gamma}) \ell-\bar{\tau} m-\tau \bar{m}, \\ \Delta n & =-(\gamma+\bar{\gamma}) n+\nu m+\bar{\nu} \bar{m}, \\ \Delta m & =\bar{\nu} \ell-\tau n+(\gamma-\bar{\gamma}) m, \\ \delta \ell & =(\bar{\alpha}+\beta) \ell-\bar{\rho} m-\sigma \bar{m}, \\ \delta n & =-(\bar{\alpha}+\beta) n+\mu m+\bar{\lambda} \bar{m}, \\ \delta m & =\bar{\lambda} \ell-\sigma n+(\beta-\bar{\alpha}) m, \\ \bar{\delta} m & =\bar{\mu} \ell-\rho n+(\alpha-\bar{\beta}) m .\end{cases}
$$

Many of the spin coefficients have a transparent geometric interpretation. Some important ones for us are: the real parts of $\rho$ and $\mu$ are the expansion of $\ell$ and $n$ respectively; $\sigma$ and $\lambda$ are the shears of $\ell$ and $n$ respectively; the vanishing of $\kappa$ and $\nu$ implies that $\ell$ and $n$ are respectively geodesic; $\epsilon+\bar{\epsilon}$ and $\gamma+\bar{\gamma}$ are respectively the

accelerations of $\ell$ and $n, \alpha-\bar{\beta}$ yields the connection in the $m-\bar{m}$ plane and thus the curvature of the manifold spanned by $m-\bar{m}$. 
The spacetime in the neighborhood of a general isolated black hole

Since the null tetrad is typically not a coordinate basis, the above definitions of the spin coefficients lead to non-trivial commutation relations:

$$
\begin{cases}(\Delta D-D \Delta) f & =(\epsilon+\bar{\epsilon}) \Delta f+(\gamma+\bar{\gamma}) D f-(\bar{\tau}+\pi) \delta f-(\tau+\bar{\pi}) \bar{\delta} f \\ (\delta D-D \delta) f & =(\bar{\alpha}+\beta-\bar{\pi}) D f+\kappa \Delta f-(\bar{\rho}+\epsilon-\bar{\epsilon}) \delta f-\sigma \bar{\delta} f \\ (\delta \Delta-\Delta \delta) f & =-\bar{\nu} D f+(\tau-\bar{\alpha}-\beta) \Delta f+(\mu-\gamma+\bar{\gamma}) \delta f+\bar{\lambda} \bar{\delta} f \\ (\bar{\delta} \delta-\delta \bar{\delta}) f & =(\bar{\mu}-\mu) D f+(\bar{\rho}-\rho) \Delta f+(\alpha-\bar{\beta}) \delta f-(\bar{\alpha}-\beta) \bar{\delta} f\end{cases}
$$

The Weyl tensor $C_{a b c d}$ breaks down into 5 complex scalars

$$
\left\{\begin{array}{l}
\Psi_{0}=C_{a b c d} \ell^{a} m^{b} \ell^{c} m^{d}, \quad \Psi_{1}=C_{a b c d} \ell^{a} m^{b} \ell^{c} n^{d}, \quad \Psi_{2}=C_{a b c d} \ell^{a} m^{b} \bar{m}^{c} n^{d}, \\
\Psi_{3}=C_{a b c d} \ell^{a} n^{b} \bar{m}^{c} n^{d}, \quad \Psi_{4}=C_{a b c d} \bar{m}^{a} n^{b} \bar{m}^{c} n^{d} .
\end{array}\right.
$$

Similarly, the Ricci tensor is decomposed into 4 real and 3 complex scalars $\Phi_{i j}$ :

$$
\left\{\begin{array}{l}
\Phi_{00}=\frac{1}{2} R_{a b} \ell^{a} \ell^{b}, \quad \Phi_{11}=\frac{1}{4} R_{a b}\left(\ell^{a} n^{b}+m^{a} \bar{m}^{b}\right), \quad \Phi_{22}=\frac{1}{2} R_{a b} n^{a} n^{b}, \quad \Lambda=\frac{R}{24}, \\
\Phi_{01}=\frac{1}{2} R_{a b} \ell^{a} m^{b}, \quad \Phi_{02}=\frac{1}{2} R_{a b} m^{a} m^{b}, \quad \Phi_{12}=\frac{1}{2} R_{a b} m^{a} n^{b}, \quad \bar{\Phi}_{i j}=\Phi_{j i} .
\end{array}\right.
$$

The 6 components of the Maxwell 2-form $\mathbf{F}_{a b}$ are written in terms of 3 complex scalars

$$
\phi_{0}=-\mathbf{F}_{a b} \ell^{a} m^{b}, \quad \phi_{1}=\frac{1}{2} \mathbf{F}_{a b}\left(n^{a} \ell^{b}+m^{a} \bar{m}^{b}\right), \quad \phi_{2}=\mathbf{F}_{a b} n^{a} \bar{m}^{b} .
$$

The source free Maxwell equations $d \mathbf{F}=0$ and $d \star \mathbf{F}=0$ are written as 4 complex scalar equations

$$
\left\{\begin{array}{l}
D \phi_{1}-\bar{\delta} \phi_{0}=(\pi-2 \alpha) \phi_{0}+2 \rho \phi_{1}-\kappa \phi_{2} \\
D \phi_{2}-\bar{\delta} \phi_{1}=-\lambda \phi_{0}+2 \pi \phi_{1}+(\rho-2 \epsilon) \phi_{2} \\
\Delta \phi_{0}-\delta \phi_{1}=(2 \gamma-\mu) \phi_{0}-2 \tau \phi_{1}+\sigma \phi_{2} \\
\Delta \phi_{1}-\delta \phi_{2}=\nu \phi_{0}-2 \mu \phi_{1}+(2 \beta-\tau) \phi_{2}
\end{array}\right.
$$

The stress energy tensor for the Maxwell field is given by

$$
T_{a b}=\frac{1}{4 \pi}\left(\mathbf{F}_{a c} \mathbf{F}_{b}{ }^{c}-\frac{1}{4} g_{a b} \mathbf{F}_{c d} \mathbf{F}^{c d}\right),
$$

which is seen to be trace-free. Using the Einstein equations, we see that for EinsteinMaxwell theory, the Ricci tensor components defined in Eqs. (7) have a simple expression in terms of the $\phi_{i}$ :

$$
\Phi_{i j}=2 \phi_{i} \bar{\phi}_{j}(i, j=0,1,2), \quad \Lambda=0 .
$$

The relation between the spin coefficients and the curvature components lead to the so called Newman-Penrose field equations which are a set of 16 complex first order differential equations. The Bianchi identities are written explicitly as 8 complex and 3 
real equations. See [28, 29, 23] for the full set of field equations and Bianchi identities. We shall later write these equations after imposing gauge and coordinate conditions.

It will also be useful to use the notion of spin weights and the op operator for derivatives in the $m-\bar{m}$ plane (which will be angular derivatives in our case). A tensor $X$ projected on the $m-\bar{m}$ plane is said to have spin weight $s$ if under a spin rotation $m \rightarrow e^{i \psi} m$, it transforms as $X \rightarrow e^{i s \psi} X$. Thus, $m^{a}$ itself has spin weight +1 while $\bar{m}^{a}$ has weight -1 . For a scalar $X=m^{a_{1}} \cdots m^{a_{p}} \bar{m}^{b_{1}} \cdots \bar{m}^{b_{q}} X_{a_{1} \cdots b_{q}}$, i.e. it has $p$ contractions with $m$ and $q$ with $\bar{m}$, then $X$ has spin weight $s=p-q$. For example, the Weyl tensor component $\Psi_{k}$ has spin weight $2-k$. Similarly, the Maxwell field component $\phi_{k}$ has weight $1-k$.

The $\bar{\partial}$ and $\bar{\varnothing}$ operators are defined as

$$
\begin{aligned}
& \text { ळ } X=m^{a_{1}} \cdots m^{a_{p}} \bar{m}^{b_{1}} \cdots \bar{m}^{b_{q}} \delta X_{a_{1} \cdots b_{q}}, \\
& \bar{\partial} X=m^{a_{1}} \cdots m^{a_{p}} \bar{m}^{b_{1}} \cdots \bar{m}^{b_{q}} \bar{\delta} X_{a_{1} \cdots b_{q}} .
\end{aligned}
$$

From Eqs. (4ii) and (4j), after projecting on to the $m-\bar{m}$ plane, we get

$$
\delta m^{a}=(\beta-\bar{\alpha}) m^{a}, \quad \bar{\delta} m^{a}=(\alpha-\bar{\beta}) m^{a} .
$$

A short calculation shows that

$$
\widetilde{\partial} X=\delta X+s(\bar{\alpha}-\beta) X, \quad \overline{\mathrm{\partial}} X=\bar{\delta} X-s(\alpha-\bar{\beta}) X .
$$

It is clear that $\check{\partial}$ and $\bar{\partial}$ act as spin raising and lowering operators. See [30] for further properties of the $\partial$ operator and its connection to representations of the rotation group.

The transformations of the null tetrad which preserve the metric are

(i) the boosts:

$$
l \rightarrow A l, \quad n \rightarrow A^{-1} n, \quad m \rightarrow m,
$$

(ii) the spin transformations in the $m-\bar{m}$ plane:

$$
m \rightarrow e^{i \psi} m, \quad \ell \rightarrow \ell, \quad n \rightarrow n
$$

(iii) the null rotations around $\ell$ :

$$
\ell \rightarrow \ell, \quad m \rightarrow m+a \ell, \quad n \rightarrow n+\bar{a} m+a \bar{m}+|a|^{2} \ell,
$$

(iv) the null rotations around $n$ (obtained by interchanging $\ell$ and $n$ in Eq. (18)) .

Again, we refer to [28, 29, 23] for a more complete discussion.

\subsection{Non-expanding and weakly isolated horizons}

A black hole in equilibrium with its surroundings is modeled quasi-locally as an isolated horizon. The basic geometrical object is a smooth 3-dimensional null surface $\Delta$ (which shall be the black hole horizon) in a Lorentzian spacetime $\left(\mathcal{M}, g_{a b}\right)$. If $\ell$ is any null normal of $\Delta$, then it must be geodesic so that $\ell^{a} \nabla_{a} \ell^{b}=\tilde{\kappa}_{(\ell)} \ell^{b}$; the acceleration $\tilde{\kappa}_{(\ell)}$ is the surface gravity associated with $\ell^{a}$. We shall consider only non-extremal horizons here, i.e. we shall always have non-vanishing $\tilde{\kappa}$. The spacetime metric $g_{a b}$ induces a 
degenerate metric on $\Delta$ which we denote $q_{a b}$ which has signature $(0++)$. Its (nonunique) inverse will be denoted $q^{a b}$. There is a volume element ${ }^{2} \epsilon_{a b}$ on $\Delta$, satisfying $\ell^{a}{ }^{2} \epsilon_{a b}=0$, which measures the area of spacelike cross-sections of $\Delta$.

A smooth 3-dimensional null surface $\Delta$ is said to be a non-expanding horizon if:

- $\Delta$ has topology $S^{2} \times \mathbb{R}$.

- The expansion $\Theta_{(\ell)}:=q^{a b} \nabla_{a} \ell_{b}$ of any null normal $\ell^{a}$ of $\Delta$ vanishes.

- The Einstein field equations hold at $\Delta$, and the matter stress-energy tensor $T_{a b}$ is such that for any future directed null-normal $\ell^{a},-T_{b}^{a} \ell^{b}$ is future causal.

We shall consider only null tetrads adapted to $\Delta$ such that, at the horizon, $\ell^{a}$ coincides with a null-normal to $\Delta$. We shall also consider a foliation of the horizon by spacelike spheres $S_{v}$ with $v$ a coordinate on the horizon which is also an affine parameter along $\ell: \mathcal{L}_{\ell} v=1$. Null rotations about $\ell^{a}$ correspond to changing the foliation.

This deceptively simple definition of a non-expanding horizon leads to a number of important results which we state here without proof (though some of these will be rederived later):

(i) The Weyl tensor components $\Psi_{0}$ and $\Psi_{1}$ vanish on the horizon. This implies that $\Psi_{2}$ is an invariant on $\Delta$ as long as the null-tetrad is adapted to the horizon; it is automatically invariant under boosts and spin rotations (it has spin weight 0), and it is invariant under null rotations around $\ell$ because $\Psi_{0}$ and $\Psi_{1}$ vanish. Similarly, the Maxwell field component $\phi_{0}$ vanishes on the horizon, and $\phi_{1}$ is invariant on $\Delta$. Both $\Psi_{2}$ and $\phi_{1}$ are also time independent on the horizon.

(ii) For a general null sub-manifold, there is no unique derivative operator compatible with the metric, and the pull back of the spacetime derivative operator $\nabla_{a}$ does not necessarily induce a connection on the hypersurface. For non-expanding horizons however, the spacetime connection does induce a unique derivative operator compatible with $q_{a b}$. Furthermore, there exists a 1-form $\omega_{a}$ such that, for any vector field $X^{a}$ tangent to $\Delta$,

$$
X^{a} \nabla_{a} \ell^{b}=X^{a} \omega_{a} \ell^{b} .
$$

The 1-form $\omega_{a}$ plays a fundamental role in what follows. The pullback of $\omega_{a}$ to the cross-sections $S$ will be denoted $\tilde{\omega}_{a}$.

(iii) The surface gravity of $\ell$ is

$$
\tilde{\kappa}_{(\ell)}=\ell^{a} \omega_{a}
$$

The curl and divergence of $\omega$ carry important physical information. The curl is related to the imaginary part of the Weyl tensor on the horizon

$$
d \omega=\operatorname{Im}\left[\Psi_{2}\right]^{2} \epsilon .
$$

and its divergence specifies the foliation of $\Delta$ by spheres [6]. 
(iv) The horizon angular momentum is well defined in the case when there is an axial symmetry $\varphi^{a}$ on $\Delta$ which preserves $q_{a b}, \omega_{a}$ and the electromagnetic field on the horizon [5]. The angular momentum is given by

$$
J=-\frac{1}{4 \pi} \oint_{S} f \operatorname{Im}\left[\Psi_{2}\right]^{2} \epsilon+\frac{1}{2 \pi} \oint_{S} g \operatorname{Im}\left[\phi_{1}\right]^{2} \epsilon,
$$

where $f$ and $g$ are respectively defined via $\varphi^{a 2} \epsilon_{a b}=\partial_{b} f$ and $\varphi^{a} \star \mathbf{F}_{a b}=\partial_{b} g$. Similarly, the electric and magnetic charges of the horizon are defined respectively as

$$
Q=\frac{1}{2 \pi} \oint_{S} \operatorname{Re}\left[\phi_{1}\right]^{2} \epsilon, \quad P=\frac{1}{2 \pi} \oint_{S} \operatorname{Im}\left[\phi_{1}\right]^{2} \epsilon .
$$

Hamiltonian methods provide a suitable notion of horizon mass [5]:

$$
M=\frac{1}{2 R} \sqrt{\left(R^{2}+Q^{2}\right)^{2}+4 J^{2}},
$$

where $R$ is the area radius of the horizon so that if $A$ is the area of the horizon crosssections then $R:=\sqrt{A / 4 \pi}$. In the case when the horizon is not exactly symmetric, one could attempt to find an approximate symmetry to replace $\varphi^{a}$ in the above equations [31, 32, 33]. It is also possible to define source multipole moments for an axisymmetric charged isolated horizon [34].

We need to strengthen the conditions of a non-expanding horizon for various physical situations. The minimum extra condition required for black hole thermodynamics and to have a well defined action principle with $\Delta$ as an inner boundary of a portion of spacetime, is formulated as a weakly isolated horizon [3]. This is to choose an equivalence class of null normals $[\ell]$, each related to the other by a constant re-scaling $\ell^{\prime}=c \ell$, such that

$$
\mathcal{L}_{\ell} \omega_{a}=0 .
$$

This can be shown to be equivalent to the zeroth law, i.e. $\tilde{\kappa}_{(\ell)}=\ell^{a} \omega_{a}$ is constant on the

horizon. Note that under a re-scaling $\ell^{a} \rightarrow f \ell^{a}, \omega_{a}$ transforms as $\omega_{a} \rightarrow \omega_{a}+D_{a} \ln f$ so that it is invariant under constant rescalings. This condition is sufficient to ensure a well defined action principle and to lead to a sensible notion of horizon mass and spin. Any non-expanding horizon can be made into a weakly isolated horizon by suitably scaling the null generators. Thus, the restriction to weakly isolated horizons is not a genuine physical restriction.

\section{The near-horizon coordinate system and null-tetrad}

Let us now assume that the vacuum Einstein equations hold in a neighborhood of the horizon $\Delta$. We will consider electromagnetic fields later in Sec. 8. Following [4] we introduce a coordinate system and null tetrad in the vicinity of $\Delta$ analogous to the Bondi coordinates near null infinity. See Fig. 1, Choose a particular null normal $\ell^{a}$ on $\Delta$. Let $v$ be the affine parameter along $\ell^{a}$ so that $\ell^{a} \nabla_{a} v=1$. Let $S_{v}$ denote the spheres of constant $v$. Introduce coordinates $x^{i}(i=2,3)$ on any one $S_{v}$ (call this sphere $S_{0}$ ) 
and require them to be constant along $\ell^{a}: \ell^{a} \nabla_{a} x^{i}=0$; this leads to a coordinate system $\left(v, x^{i}\right)$ on $\Delta$. Let $n^{a}$ be a future directed inward pointing null vector orthogonal to the $S_{v}$ and normalized such that $\ell \cdot n=-1$. Extend $n^{a}$ off $\Delta$ geodesically, with $r$ being an affine parameter along $-n^{a}$; set $r=0$ at $\Delta$. This yields a family of null surfaces $\mathcal{N}_{v}$ parameterized by $v$ and orthogonal to the spheres $S_{v}$. Set $\left(v, x^{i}\right)$ to be constant along the integral curves of $n^{a}$ to obtain a coordinate system $\left(v, r, x^{i}\right)$ in a neighborhood of $\Delta$. Choose a complex null vector $m^{a}$ tangent to $S_{0}$. Lie drag $m^{a}$ along $\ell^{a}$ :

$$
\mathcal{L}_{\ell} m^{a}=0 \quad \text { on } \Delta .
$$

We thus obtain a null tetrad $(\ell, n, m, \bar{m})$ on $\Delta$. Finally, parallel transport $\ell$ and $m$ along $-n^{a}$ to obtain a null tetrad in the neighborhood of $\Delta$. This construction is fixed up to the choice of the $x^{i}$ and $m^{a}$ on an initial cross-section $S_{0}$. We are allowed to perform an arbitrary spin transformation $m \rightarrow e^{i \psi} m$ on $S_{0}$.

It is easy to write the elements null tetrad in these $\left(v, r, x^{i}\right)$ coordinates. Let us start with $n_{a}$ and $n^{a}$. We have the family of null surfaces $\mathcal{N}_{v}$ parameterized by $v ; n_{a}$ is normal to the $\mathcal{N}_{v}$, and $r$ is an affine parameter along $-n^{a}$. This implies that we can choose

$$
n_{a}=-\partial_{a} v \quad \text { and } \quad n^{a} \nabla_{a}:=\Delta=-\frac{\partial}{\partial r} .
$$

To satisfy the inner-product relations, the other basis vectors must be of the form:

$$
\ell^{a} \nabla_{a}:=D=\frac{\partial}{\partial v}+U \frac{\partial}{\partial r}+X^{i} \frac{\partial}{\partial x^{i}}, \quad m^{a} \nabla_{a}:=\delta=\Omega \frac{\partial}{\partial r}+\xi^{i} \frac{\partial}{\partial x^{i}} .
$$

The frame functions $U, X^{i}$ are real while $\Omega, \xi^{i}$ are complex. We want $\ell^{a}$ to be a null normal of $\Delta$ so that the null tetrad is adapted to the horizon. Since $\partial_{v}$ is tangent to the null generators of $\Delta$, this clearly requires that $U, X^{i}$ must vanish on the horizon. Similarly, we want $m^{a}$ to be tangent to the spheres $S_{v}$ at the horizon, so $\Omega$ should also vanish on $\Delta$. Thus, $U, X^{i}, \Omega$ are all $\mathcal{O}(r)$ functions.

We expand the spin coefficients, Weyl tensor components and the directional derivatives in a power series in $r$ away from the horizon

$$
X=X^{(0)}+r X^{(1)}+\frac{1}{2} r^{2} X^{(2)} \cdots
$$

Thus, for example we will have

$$
\Psi_{k}=\Psi_{k}^{(0)}+r \Psi_{k}^{(1)}+\frac{1}{2} r^{2} \Psi_{k}^{(2)}+\ldots
$$

The same notation will be used for the frame fields $U$ and $\Omega$. However, for the frame fields $X^{i}$ and $\xi^{i}$, we will write

$$
\xi^{i}=\xi_{(0)}^{i}+r \xi_{(1)}^{i}+\frac{1}{2} r^{2} \xi_{(2)}^{i}+\ldots
$$

To avoid clutter we shall not be completely consistent with this notation. Thus, we shall not use any index for the spin weighted angular derivative $\precsim$; it is to be understood that $\widetilde{\partial}$ always refers to $ð^{(0)}$ in this paper. Similarly, where we don't expect any confusion, we shall often drop the index on the directional derivatives such as $D$ and $\delta$; in this case, 
unless mentioned otherwise, the relevant order of the operator is the same as the order of the operand. For example, $\delta \Psi_{2}^{(0)}$ refers to $\delta^{(0)} \Psi_{2}^{(0)}$.

The variables we need to solve for are the frame fields $U, X^{i}, \Omega, \xi^{i}$, the 12 spin coefficients and the Weyl tensor components $\Psi_{k}$. The equations are the commutations relations Eqs. (28), the 16 field equations and 8 of the 11 Bianchi identities. Three of the Bianchi identities involve only the Ricci tensor, thus they will need to be considered when matter fields are present. In Sec. 8 we will consider the Maxwell equations and the 3 additional Bianchi identities as well. All these are first order differential equations and each of these sets of equations has possibly three kinds of equations: evolution equations which involve derivatives along $v$, i.e. $D$, and do not contain any radial derivatives $\Delta$, equations which contain only purely angular derivatives $\delta$ and $\bar{\delta}$, and finally the radial equations involving $\Delta$. In order to integrate these equations, we proceed as follows. We start with suitable data on some initial cross section $S_{0}$ of the horizon and use the non-radial equations to propagate them at all points of the horizon. Starting with this horizon data and the appropriate data on the past light cone $\mathcal{N}_{0}$ containing $S_{0}$, the radial equations then yield the first radial derivatives and, iteratively, all successive higher derivatives as well. At each step, we will need to expand the non-radial equations in powers of $r$, and ensure that we have consistency order-by-order in $r$. We now carry out this procedure in detail and spell out the free data.

We start with the conditions on the spin coefficients. Since $n^{a}$ is an affinely parameterized geodesic, and $\ell$ and $m$ are parallel propagated along $n^{a}$, we have $\Delta n=\Delta \ell=\Delta m=0$. From Eqs. (4d), (4e) and (4f) , this leads to

$$
\gamma=\tau=\nu=0 \text {. }
$$

Imposing Eq. (32) in the commutation relations (5) leads to

$$
\begin{cases}(\Delta D-D \Delta) f & =(\epsilon+\bar{\epsilon}) \Delta f-\pi \delta f-\bar{\pi} \bar{\delta} f \\ (\delta D-D \delta) f & =(\bar{\alpha}+\beta-\bar{\pi}) D f+\kappa \Delta f-(\bar{\rho}+\epsilon-\bar{\epsilon}) \delta f-\sigma \bar{\delta} f \\ (\delta \Delta-\Delta \delta) f & =-(\bar{\alpha}+\beta) \Delta f+\mu \delta f+\bar{\lambda} \bar{\delta} f \\ (\bar{\delta} \delta-\delta \bar{\delta}) f & =(\bar{\mu}-\mu) D f+(\bar{\rho}-\rho) \Delta f+(\alpha-\bar{\beta}) \delta f-(\bar{\alpha}-\beta) \bar{\delta} f\end{cases}
$$

Substituting $f=v$ yields

$$
\pi=\alpha+\bar{\beta}, \quad \mu=\bar{\mu} .
$$

Eqs. (32) and (34) are the basic conditions on the spin coefficients which hold at all spacetime points where the coordinate system is valid. In addition, we have the boundary condition at the horizon, namely that $\ell$ is geodesic, normal to a smooth surface, and expansion free. This implies

$$
\rho^{(0)}=\kappa^{(0)}=0 \text {. }
$$

The subscript ${ }^{(0)}$ indicates that these are the values at the horizon, i.e. at $r=0$. From the Raychaudhuri equation applied to the null generators of the horizon, we get

$$
D \rho^{(0)}-\bar{\delta} \kappa^{(0)}=\left(\rho^{(0)}\right)^{2}+\left|\sigma^{(0)}\right|^{2}+\left(\epsilon^{(0)}+\bar{\epsilon}^{(0)}\right) \rho^{(0)}-2 \alpha^{(0)} \kappa^{(0)}
$$


(this is also one of the Newman-Penrose field equations considered later). This leads to $\sigma^{(0)}=0$. In addition, we can make a spin transformation on $S_{0}$ and set

$$
\epsilon^{(0)}-\bar{\epsilon}^{(0)}=0 \text {. }
$$

From Eq. (33b) and the above conditions on the spin coefficients at the horizon, this also ensures that $\mathcal{L}_{\ell} m^{a}=0$ at the horizon. Finally, we assume that we have scaled the null normal appropriately (i.e. we have chosen the coordinate $v$ and the foliation $S_{v}$ ) so that the horizon is weakly isolated: $\mathcal{L}_{\ell} \omega_{a}=0$. In terms of the Newman-Penrose spin coefficients, $\omega_{a}$ is written as

$$
\omega_{a}=-n_{b} \nabla_{a} \ell^{b}=(\epsilon+\bar{\epsilon}) n_{a}+\pi^{(0)} m_{a}+\bar{\pi}^{(0)} \bar{m}_{a} .
$$

Thus, $\Delta$ will be a weakly isolated horizon if we choose

$$
\tilde{\kappa}:=\epsilon^{(0)}+\bar{\epsilon}^{(0)}=\text { constant }, \quad D \pi^{(0)}=0 .
$$

Among the free functions that we need to specify, we can choose a constant for the surface gravity (perhaps determined by the mass and spin [5, 3]), and a function $\pi^{(0)}$ on the initial cross-section $S_{0}$.

\section{The reduced system of equations}

With the conditions Eqs. (32) and (34) on the spin coefficients at hand, we now impose them in the commutator relations, the field equations and the Bianchi identities. The functions $U, X^{i}, \Omega, \xi^{i}$ are determined by the commutation relations (33) by substituting, in turn, $r$ and $x^{i}$ for $f$, and imposing Eqs. (32) and (34) on the spin coefficients. First the radial derivatives:

$$
\begin{cases}\Delta U & =-(\epsilon+\bar{\epsilon})-\pi \Omega-\bar{\pi} \bar{\Omega} \\ \Delta X^{i} & =-\pi \xi^{i}-\bar{\pi} \bar{\xi}^{i} \\ \Delta \Omega & =-\bar{\pi}-\mu \Omega-\bar{\lambda} \bar{\Omega} \\ \Delta \xi^{i} & =-\mu \xi^{i}-\bar{\lambda} \bar{\xi}^{i}\end{cases}
$$

The propagation equations along $v$ are

$$
\begin{cases}D \Omega-\delta U & =\kappa+\rho \Omega+\sigma \bar{\Omega} \\ D \xi^{i}-\delta X^{i} & =\bar{\rho} \xi^{i}+\sigma \bar{\xi}^{i} .\end{cases}
$$

Let us now turn to the field equations. After imposing Eqs. (32) and (34) on the spin coefficients and ignoring matter terms for now, the field equations involving radial 
The spacetime in the neighborhood of a general isolated black hole

derivatives are:

$$
\left\{\begin{array}{l}
\Delta \lambda=-2 \lambda \mu-\Psi_{4}, \\
\Delta \mu=-\mu^{2}-|\lambda|^{2} \\
\Delta \rho=-\mu \rho-\sigma \lambda-\Psi_{2}, \\
\Delta \sigma=-\mu \sigma-\bar{\lambda} \rho . \\
\Delta \kappa=-\bar{\pi} \rho-\pi \sigma-\Psi_{1}, \\
\Delta \epsilon=-\bar{\pi} \alpha-\pi \beta-\Psi_{2}, \\
\Delta \pi=-\pi \mu-\bar{\pi} \lambda-\Psi_{3} \\
\Delta \beta=-\mu \beta-\alpha \bar{\lambda} \\
\Delta \alpha=-\beta \lambda-\mu \alpha-\Psi_{3} .
\end{array}\right.
$$

The time evolution equations become:

$$
\left\{\begin{array}{l}
D \rho-\bar{\delta} \kappa=\rho^{2}+|\sigma|^{2}+(\epsilon+\bar{\epsilon}) \rho-2 \alpha \kappa \\
D \sigma-\delta \kappa=(\rho+\bar{\rho}+2 \epsilon) \sigma-2 \beta \kappa+\Psi_{0} \\
D \alpha-\bar{\delta} \epsilon=(\rho-\epsilon) \alpha+\beta \bar{\sigma}-\bar{\beta} \epsilon-\kappa \lambda+(\epsilon+\rho) \pi \\
D \beta-\delta \epsilon=(\alpha+\pi) \sigma+(\bar{\rho}-\epsilon) \beta-\mu \kappa-(\bar{\alpha}-\bar{\pi}) \epsilon+\Psi_{1} \\
D \lambda-\bar{\delta} \pi=(\rho-2 \epsilon) \lambda+\bar{\sigma} \mu+2 \alpha \pi \\
D \mu-\delta \pi=(\bar{\rho}-2 \epsilon) \mu+\sigma \lambda+2 \beta \pi+\Psi_{2} .
\end{array}\right.
$$

The angular field equations are:

$$
\left\{\begin{array}{l}
\delta \rho-\bar{\delta} \sigma=\bar{\pi} \rho-(3 \alpha-\bar{\beta}) \sigma-\Psi_{1} \\
\delta \alpha-\bar{\delta} \beta=\mu \rho-\lambda \sigma+|\alpha|^{2}+|\beta|^{2}-2 \alpha \beta-\Psi_{2} \\
\delta \lambda-\bar{\delta} \mu=\pi \mu+(\bar{\alpha}-3 \beta) \lambda-\Psi_{3}
\end{array}\right.
$$

Finally, we have the Bianchi identities which, in the NP formalism, are written as a set of nine complex and two real equations; in the absence of matter, only 8 complex equations survive. The radial Bianchi identities reduce to:

$$
\left\{\begin{array}{l}
\Delta \Psi_{0}-\delta \Psi_{1}=-\mu \Psi_{0}-2 \beta \Psi_{1}+3 \sigma \Psi_{2} \\
\Delta \Psi_{1}-\delta \Psi_{2}=-2 \mu \Psi_{1}+2 \sigma \Psi_{3} \\
\Delta \Psi_{2}-\delta \Psi_{3}=-3 \mu \Psi_{2}+2 \beta \Psi_{3}+\sigma \Psi_{4} \\
\Delta \Psi_{3}-\delta \Psi_{4}=-4 \mu \Psi_{3}+4 \beta \Psi_{4}
\end{array}\right.
$$

Note that there is no equation for the radial derivative of $\Psi_{4}$. Among all the fields that we are solving for, this is in fact the only one for which this happens. This means that $\Psi_{4}$ (in this case, its radial derivatives) is the free data that must be specified on the null cone $\mathcal{N}_{0}$ originating from $S_{0}$. 
The spacetime in the neighborhood of a general isolated black hole

Finally, we have the components of the Bianchi equations for evolution of the Weyl tensor components:

$$
\left\{\begin{array}{l}
D \Psi_{1}-\bar{\delta} \Psi_{0}=(\pi-4 \alpha) \Psi_{0}+2(2 \rho+\epsilon) \Psi_{1}-3 \kappa \Psi_{2} \\
D \Psi_{2}-\bar{\delta} \Psi_{1}=-\lambda \Psi_{0}-2(\pi-\alpha) \Psi_{1}+3 \rho \Psi_{2}-2 \kappa \Psi_{3} \\
D \Psi_{3}-\bar{\delta} \Psi_{2}=-2 \lambda \Psi_{1}+3 \pi \Psi_{2}+2(\rho-\epsilon) \Psi_{3}-\kappa \Psi_{4} \\
D \Psi_{4}-\bar{\delta} \Psi_{3}=-3 \lambda \Psi_{2}+2(\alpha+2 \pi) \Psi_{3}+(\rho-4 \epsilon) \Psi_{4}
\end{array}\right.
$$

Having written down the full set of differential equations, we are now ready to solve them locally near the horizon order-by-order in $r$.

\section{The intrinsic horizon geometry}

We first consider the intrinsic geometry of the horizon contained in the time evolution equations at the horizon, i.e. the $\mathcal{O}\left(r^{0}\right)$ part of Eqs. (43). This has been studied elsewhere in great detail [6, 3, 7, so we shall be brief. We already have that $\ell^{a}$ is geodesic so that $\kappa^{(0)}=0$, and the expansion and twist of $\ell$ vanish, which implies $\rho^{(0)}=0$. The Eqs. (43) in turn lead to

$$
\left\{\begin{array}{l}
\sigma^{(0)}=0, \quad \Psi_{0}^{(0)}=0 \\
D \alpha^{(0)}-\bar{\delta} \epsilon^{(0)}=\epsilon^{(0)} \alpha^{(0)}-\bar{\beta}^{(0)} \epsilon^{(0)}+\epsilon^{(0)} \pi^{(0)} \\
D \beta^{(0)}-\delta \epsilon^{(0)}=\left(\alpha^{(0)}+\pi^{(0)}\right) \sigma^{(0)}+\epsilon^{(0)} \beta^{(0)}-\left(\bar{\alpha}^{(0)}-\bar{\pi}^{(0)}\right) \epsilon^{(0)}+\Psi_{1}^{(0)} \\
D \lambda^{(0)}-\bar{\delta} \pi^{(0)}=-2 \epsilon^{(0)} \lambda^{(0)}+2 \alpha^{(0)} \pi^{(0)} \\
D \mu^{(0)}-\delta \pi^{(0)}=-2 \epsilon^{(0)} \mu^{(0)}+2 \beta^{(0)} \pi^{(0)}+\Psi_{2}^{(0)}
\end{array}\right.
$$

Adding Eq. (47b) to the complex conjugate of Eq. (47c), and using the conditions on the spin coefficients leads to

$$
D \alpha^{(0)}=0, \quad D \beta^{(0)}=0, \quad \Psi_{1}^{(0)}=0 .
$$

The remaining two equations tell us about the time dependence of the expansion and shear of $n$ at the horizon:

$$
\left\{\begin{array}{l}
D \lambda^{(0)}+\tilde{\kappa} \lambda^{(0)}=\bar{\delta} \pi^{(0)}+2 \alpha^{(0)} \pi^{(0)} \\
D \mu^{(0)}+\tilde{\kappa} \mu^{(0)}=\delta \pi^{(0)}+2 \beta^{(0)} \pi^{(0)}+\Psi_{2}^{(0)}
\end{array}\right.
$$

Using the operator for the angular derivatives, and noting that $\pi$ has spin weight -1 , this is written more cleanly as

$$
\left\{\begin{array}{l}
D \lambda^{(0)}+\tilde{\kappa} \lambda^{(0)}=\overline{\overline{\mathrm{d}}} \pi^{(0)}+\left(\pi^{(0)}\right)^{2} \\
D \mu^{(0)}+\tilde{\kappa} \mu^{(0)}=\overline{\mathrm{d}} \pi^{(0)}+\left|\pi^{(0)}\right|^{2}+\Psi_{2}^{(0)}
\end{array}\right.
$$


These two equations can be shown to be identical to Eq. (3.9) of [6] with the matter terms set to zero.

Now consider the angular field equations (44) at the horizon. The first of these leads to $\Psi^{(0)}=0$ which we already knew. Eq. (44c) gives

$$
\Psi_{3}^{(0)}=\overline{\mathrm{\partial}} \mu^{(0)}+\pi^{(0)} \mu^{(0)}-\breve{\partial} \lambda^{(0)}-\bar{\pi}^{(0)} \lambda^{(0)} .
$$

The real and imaginary parts of Eq. (44b) give respectively

$$
\begin{aligned}
-2 \operatorname{Re} \Psi_{2}^{(0)} & =\delta a+\bar{\delta} \bar{a}-2|a|^{2}, \quad a:=\alpha^{(0)}-\bar{\beta}^{(0)}, \\
-2 i \operatorname{Im} \Psi_{2}^{(0)} & =\partial \pi^{(0)}-\bar{\partial} \bar{\pi}^{(0)} .
\end{aligned}
$$

Since $a=\alpha^{(0)}-\bar{\beta}^{(0)}$ determines the connection on the horizon cross-section, it's derivative must be connected with the scalar 2-curvature of the cross-section. In fact, it is not hard to show that the first equation is equivalent to

$$
{ }^{2} \tilde{\mathcal{R}}=-4 \operatorname{Re} \Psi_{2}^{(0)}
$$

The second equation relates the curl of $\tilde{\omega}$ with the imaginary part of $\Psi_{2}$ and thus reproduces Eq. (21).

Now the Bianchi identities Eqs. (46) lead to

$$
\left\{\begin{array}{l}
D \Psi_{2}^{(0)}=0 \\
D \Psi_{3}^{(0)}+\tilde{\kappa} \Psi_{3}^{(0)}=\bar{\delta} \Psi_{2}^{(0)}+3 \pi^{(0)} \Psi_{2}^{(0)} \\
D \Psi_{4}^{(0)}+2 \tilde{\kappa} \Psi_{4}^{(0)}=\bar{\delta} \Psi_{3}^{(0)}+5 \pi^{(0)} \Psi_{3}^{(0)}-3 \lambda^{(0)} \Psi_{2}^{(0)}
\end{array}\right.
$$

The second of these can be shown to follow from Eq. (52), so it doesn't yield any new information, but the last equation gives the time evolution of $\Psi_{4}$ at the horizon.

To summarize, here is the free data required at the horizon. We start with a choice of null generator $\ell^{a}$ and a constant surface gravity $\tilde{\kappa}:=\epsilon^{(0)}+\bar{\epsilon}^{(0)}$, and an affine parameter $v$. Surfaces of constant $v$ are spheres $S_{v}$, and we choose a basis $m, \bar{m}$ tangent to the $S_{v}$ and Lie dragged along $\ell^{a}$. Then, on any one of the spheres, say $S_{0}$, choose the transversal expansion and shear $\mu^{(0)}, \lambda^{(0)}$, the spin coefficient $\pi^{(0)}$, the connection on $S_{0}: \alpha^{(0)}-\bar{\beta}^{(0)}$, and the transverse gravitational radiation $\Psi_{4}^{(0)}$. The field equations then show that $\pi^{(0)}, \alpha^{(0)}-\bar{\beta}^{(0)}$ are time independent. The transversal shear and expansion $\lambda^{(0)}(v), \mu^{(0)}(v)$ satisfy Eqs. (51) which means that their time evolution is:

$$
\left\{\begin{array}{l}
\mu^{(0)}(v)=\mu^{(0)}(0) e^{-\tilde{\kappa} v}+\frac{1}{\tilde{\kappa}}\left[\bar{\partial} \pi^{(0)}+\left(\pi^{(0)}\right)^{2}\right]\left(1-e^{-\tilde{\kappa} v}\right), \\
\lambda^{(0)}(v)=\lambda^{(0)}(0) e^{-\tilde{\kappa} v}+\frac{1}{\tilde{\kappa}}\left[\check{\partial} \pi^{(0)}+\left|\pi^{(0)}\right|^{2}+\Psi_{2}^{(0)}\right]\left(1-e^{-\tilde{\kappa} v}\right) .
\end{array}\right.
$$

The Weyl tensor components $\Psi_{0}^{(0)}, \Psi_{1}^{(0)}$ vanish. $\Psi_{2}^{(0)}$ is time independent, it's real part is determined by $\alpha^{(0)}-\bar{\beta}^{(0)}$ according to Eq. (51), and its imaginary part by $\pi^{(0)}=\alpha^{(0)}+\bar{\beta}^{(0)}$ according to Eq. (54); $\Psi_{3}^{(0)}$ is determined from Eq. (152) and its time evolution is of the same form as for $\lambda^{(0)}, \mu^{(0)}$. From Eq. (46b) we get

$$
\Psi_{3}^{(0)}(v)=\Psi_{3}^{(0)}(0) e^{-\tilde{\kappa} v}+\frac{1}{\tilde{\kappa}}\left[\overline{\widetilde{\partial}} \Psi_{2}^{(0)}+3 \pi^{(0)} \Psi_{2}^{(0)}\right]\left(1-e^{-\tilde{\kappa} v}\right) .
$$


The spacetime in the neighborhood of a general isolated black hole

Finally, $\Psi_{4}^{(0)}$ can be freely specified on $S_{0}$ and it evolves in time according to Eq. (156c); this leads to a time dependence of the form

$$
\Psi_{4}^{(0)}(v)=\Psi_{4}^{(0)}(0)+\frac{A}{2 \tilde{\kappa}}\left(1-e^{-2 \tilde{\kappa} v}\right)+\frac{B}{\tilde{\kappa}} e^{-\tilde{\kappa} v}\left(1-e^{-\tilde{\kappa} v}\right)
$$

where the time independent angular functions $A$ and $B$ are defined via

$$
\begin{aligned}
& \left(\overline{\bar{\partial}}+5 \pi^{(0)}\right) \Psi_{3}^{(0)}-3 \lambda^{(0)} \Psi_{2}^{(0)}=A+B e^{-\tilde{\kappa} v}, \\
& A=\frac{1}{\tilde{\kappa}}\left[\left(\overline{\check{\partial}}+5 \pi^{(0)}\right)\left(\overline{\mathrm{\partial}}+3 \pi^{(0)}\right)-3\left(\overline{\mathrm{\partial}} \pi^{(0)}+\left|\pi^{(0)}\right|^{2}+\Psi_{2}^{(0)}\right)\right] \Psi_{2}^{(0)}, \\
& B=\left(\overline{\mathrm{\partial}}+5 \pi^{(0)}\right) \Psi_{3}^{(0)}(0)-3 \lambda^{(0)} \Psi_{2}^{(0)}-A .
\end{aligned}
$$

We note that the time evolution on an extremal horizon $(\tilde{\kappa}=0)$ would be very different; see e.g. [6, 7]. As we shall see in the next section, not only can we specify $\Psi_{4}$ freely on $S_{0}$, but also all of its radial derivatives. The free data on the transversal null surface $\mathcal{N}_{0}$ is in fact $\Psi_{4}$.

\section{The radial equations}

With the intrinsic horizon geometry understood, we turn our attention to the radial equations (42) and (45) which determine all radial derivatives for all the non-zero spin coefficients and Weyl tensor components. The first radial derivatives are easily obtained by substituting the horizon values on the right hand sides of Eqs. (42) and (45)):

$$
\left\{\begin{array}{l}
\kappa^{(1)}=\sigma^{(1)}=0 \\
\rho^{(1)}=\Psi_{2}^{(0)} \\
\epsilon^{(1)}+\bar{\epsilon}^{(1)}=2\left|\pi^{(0)}\right|^{2}+2 \operatorname{Re}\left[\Psi_{2}^{(0)}\right] \\
\epsilon^{(1)}-\bar{\epsilon}^{(1)}=\bar{\pi}^{(0)}\left(\alpha^{(0)}-\bar{\beta}^{(0)}\right)-\bar{\pi}^{(0)}\left(\bar{\alpha}^{(0)}-\beta^{(0)}\right)+\Psi_{2}^{(0)}-\bar{\Psi}_{2}^{(0)} \\
\lambda^{(1)}=2 \mu^{(0)} \lambda^{(0)}+\Psi_{4}^{(0)} \\
\mu^{(1)}=\left(\mu^{(0)}\right)^{2}+\left|\lambda^{(0)}\right|^{2} \\
\pi^{(1)}=\alpha^{(1)}+\bar{\beta}^{(1)}=\pi^{(0)} \mu^{(0)}+\bar{\pi}^{(0)} \lambda^{(0)}+\Psi_{3}^{(0)} \\
\alpha^{(1)}-\bar{\beta}^{(1)}=\mu^{(0)}\left(\alpha^{(0)}-\bar{\beta}^{(0)}\right)+\lambda^{(0)}\left(\beta^{(0)}-\bar{\alpha}^{(0)}\right)+\Psi_{3}^{(0)} \\
\Psi_{0}^{(1)}=0 \\
\Psi_{1}^{(1)}=-\partial \Psi_{2}^{(0)} \\
\Psi_{2}^{(1)}=-\left(ð+\bar{\pi}^{(0)}\right) \Psi_{3}^{(0)}+3 \mu^{(0)} \Psi_{2}^{(0)} \\
\Psi_{3}^{(1)}=-\left(ð+2 \bar{\pi}^{(0)}\right) \Psi_{4}^{(0)}+4 \mu^{(0)} \Psi_{3}^{(0)}
\end{array}\right.
$$

We can continue this process by applying the radial derivative $\Delta$ to Eqs. (42) once again, and substituting the first derivatives from above on the right hand side. This leads to 
expressions for the second derivatives in terms of the horizon data:

$$
\left\{\begin{array}{l}
\kappa^{(2)}=-\left(ð-\bar{\pi}^{(0)}\right) \Psi_{2}^{(0)} \\
\sigma^{(2)}=\bar{\lambda}^{(0)} \Psi_{2}^{(0)} \\
\rho^{(2)}=4 \mu^{(0)} \Psi_{2}^{(0)}-\left(ð+\bar{\pi}^{(0)}\right) \Psi_{3}^{(0)}, \\
\lambda^{(2)}=6\left(\mu^{(0)}\right)^{2} \lambda^{(0)}+2 \lambda^{(0)}\left|\lambda^{(0)}\right|^{2}+2 \mu^{(0)} \Psi_{4}^{(0)}+\Psi_{4}^{(1)}, \\
\mu^{(2)}=2\left(\mu^{(0)}\right)^{3}+6 \mu^{(0)}\left|\lambda^{(0)}\right|^{2}+\lambda^{(0)} \bar{\Psi}_{4}^{(0)}+\bar{\lambda}^{(0)} \Psi_{4}^{(0)} \\
\pi^{(2)}=2 \pi^{(0)}\left(\left(\mu^{(0)}\right)^{2}+\left|\lambda^{(0)}\right|^{2}\right)+4 \bar{\pi}^{(0)} \lambda^{(0)} \mu^{(0)}+5 \mu^{(0)} \Psi_{3}^{(0)} \\
\quad+\lambda^{(0)} \bar{\Psi}_{3}^{(0)}-\left(ð+\bar{\pi}^{(0)}\right) \Psi_{4}^{(0)}, \\
\quad+\Psi_{4}^{(0)}\left(\beta^{(0)}-\bar{\alpha}^{(0)}\right)+5 \mu^{(0)} \Psi_{3}^{(0)}-\lambda^{(0)} \bar{\Psi}_{3}^{(0)}-\left(ð+2 \bar{\pi}^{(0)}\right) \Psi_{4}^{(0)} . \\
\alpha^{(2)}-\bar{\beta}^{(2)}=2\left(\alpha^{(0)}-\bar{\beta}^{(0)}\right)\left(\left(\mu^{(0)}\right)^{2}+\left|\lambda^{(0)}\right|^{2}\right)+4 \mu^{(0)} \lambda^{(0)}\left(\bar{\alpha}^{(0)}\right) \\
\end{array}\right.
$$

Finally we can also investigate the non-radial equations to $\mathcal{O}(r)$. For this, we need the directional derivatives to $\mathcal{O}(r)$ which we have already calculated:

$$
\left\{\begin{array}{l}
\Delta=-\frac{\partial}{\partial r} \\
D=\frac{\partial}{\partial v}+r\left(\epsilon^{(0)}+\bar{\epsilon}^{(0)}\right) \frac{\partial}{\partial r}+r \pi^{(0)} \xi_{(0)}^{i} \frac{\partial}{\partial x^{i}}+r \bar{\pi}^{(0)} \bar{\xi}_{(0)}^{i} \frac{\partial}{\partial x^{i}}+\mathcal{O}\left(r^{2}\right), \\
\delta=\xi_{(0)}^{i} \frac{\partial}{\partial x^{i}}+r \mu^{(0)} \xi_{(0)}^{i} \frac{\partial}{\partial x^{i}}+r \bar{\lambda}^{(0)} \bar{\xi}_{(0)}^{i} \frac{\partial}{\partial x^{i}}+r \bar{\pi}^{(0)} \frac{\partial}{\partial r}+\mathcal{O}\left(r^{2}\right) .
\end{array}\right.
$$

We substitute these on the left hand sides of Eqs. (41), (43) and (44), and also expand the right hand sides in powers of $r$. Equating powers of $r$ on both sides yields differential equations at each order. The $\mathcal{O}\left(r^{0}\right)$ terms have already been studied in Sec. 5 since these deal with the intrinsic horizon geometry. The $\mathcal{O}(r)$ equations are straightforward in some cases. For example, Eq. (43b) is trivial because $\kappa, \sigma, \Psi_{0}$ are all $\mathcal{O}\left(r^{2}\right)$ quantities as we have already seen in Eqs. (63). Similarly, Eq. (43a) is also easy. Using $\rho=r \Psi_{2}^{(0)}+\mathcal{O}\left(r^{2}\right)$ and the vanishing of $\kappa, \sigma$ to $\mathcal{O}(r)$, we get

$$
\frac{\partial}{\partial v} \Psi_{2}^{(0)}=0
$$

which we already knew. The remaining equations are similarly straightforward, but a lot more tedious. In the end, it turns out that Eqs. (41), (43) and (44) do not yield any new information at $\mathcal{O}(r)$.

\section{The expansion of the metric}

To expand the metric in powers of $r$, we start with the frame fields defined in Eqs.(27) and (28), and the radial frame equations (40) derived from the commutation relations. The strategy is the same as for the spin coefficients. Eqs. (40) give us the first radial derivatives by substituting the horizon values on the right hand side, and taking higher derivatives leads to the higher order terms. The calculations are straightforward and 
The spacetime in the neighborhood of a general isolated black hole

lead to the following expansions:

$$
\left\{\begin{array}{l}
U=r \tilde{\kappa}+r^{2}\left(2\left|\pi^{(0)}\right|^{2}+\operatorname{Re}\left[\Psi_{2}^{(0)}\right]\right)+\mathcal{O}\left(r^{3}\right), \\
\Omega=r \bar{\pi}^{(0)}+r^{2}\left(\mu^{(0)} \bar{\pi}^{(0)}+\bar{\lambda}^{(0)} \pi^{(0)}+\frac{1}{2} \bar{\Psi}_{3}^{(0)}\right)+\mathcal{O}\left(r^{3}\right), \\
X^{i}=\bar{\Omega} \xi_{(0)}^{i}+\Omega \bar{\xi}_{(0)}^{i}+\mathcal{O}\left(r^{3}\right) \\
\xi^{i}=\left[1+r \mu^{(0)}+r^{2}\left(\left(\mu^{(0)}\right)^{2}+\left|\lambda^{(0)}\right|^{2}\right)\right] \xi_{(0)}^{i} \\
\quad+\left[r \bar{\lambda}^{(0)}+r^{2}\left(2 \mu^{(0)} \bar{\lambda}^{(0)}+\frac{1}{2} \bar{\Psi}_{4}^{(0)}\right)\right] \bar{\xi}_{(0)}^{i}+\mathcal{O}\left(r^{3}\right) .
\end{array}\right.
$$

The contravariant metric is seen to be given in terms of the frame fields as follows:

$$
\left\{\begin{array}{l}
g^{r r}=2\left(U+|\Omega|^{2}\right) \\
g^{v r}=1 \\
g^{r i}=X^{i}+\bar{\Omega} \xi^{i}+\Omega \bar{\xi}^{i} \\
g^{i j}=\xi^{i} \bar{\xi}^{j}+\bar{\xi}^{i} \xi^{j}
\end{array}\right.
$$

An explicit calculation yields

$$
\begin{aligned}
g^{r r}= & 2 \tilde{\kappa} r+2 r^{2}\left(3\left|\pi^{(0)}\right|^{2}+\operatorname{Re}\left[\Psi_{2}^{(0)}\right]\right)+\mathcal{O}\left(r^{3}\right) \\
g^{i r}= & {\left[2 r \pi^{(0)}+r^{2}\left(2 \mu^{(0)} \pi^{(0)}+2 \lambda^{(0)} \bar{\pi}^{(0)}+\frac{1}{2} \Psi_{3}^{(0)}\right)+\mathcal{O}\left(r^{3}\right)\right] \xi_{(0)}^{i} } \\
& +\left[2 r \bar{\pi}^{(0)}+r^{2}\left(2 \mu^{(0)} \bar{\pi}^{(0)}+2 \bar{\lambda}^{(0)} \pi^{(0)}+\frac{1}{2} \bar{\Psi}_{3}^{(0)}\right)+\mathcal{O}\left(r^{3}\right)\right] \bar{\xi}_{(0)}^{i} . \\
g^{i j}= & \left(1+2 r \mu^{(0)}+3 r^{2}\left(\left(\mu^{(0)}\right)^{2}+\left|\lambda^{(0)}\right|^{2}\right)\right)\left(\xi_{(0)}^{i} \bar{\xi}_{(0)}^{j}+\bar{\xi}_{(0)}^{i} \xi_{(0)}^{j}\right) \\
& +\left[r \lambda^{(0)}+r^{2}\left(3 \mu^{(0)} \lambda^{(0)}+\frac{1}{2} \Psi_{4}^{(0)}\right)\right] 2 \xi_{(0)}^{i} \xi_{(0)}^{j} \\
& +\left[r \bar{\lambda}^{(0)}+r^{2}\left(3 \mu^{(0)} \bar{\lambda}^{(0)}+\frac{1}{2} \bar{\Psi}_{4}^{(0)}\right)\right] 2 \bar{\xi}_{(0)}^{i} \bar{\xi}_{(0)}^{j}+\mathcal{O}\left(r^{3}\right) .
\end{aligned}
$$

The null co-tetrad, i.e. the dual basis for the null tetrad found above can also be calculated easily up to $\mathcal{O}\left(r^{2}\right)$ :

$$
\begin{aligned}
& n=-d v \\
& \ell=d r-\left(\tilde{\kappa} r+\operatorname{Re}\left[\Psi_{2}^{(0)}\right] r^{2}\right) d v-\left(\pi^{(0)} r+\frac{1}{2} \Psi_{3}^{(0)} r^{2}\right) \xi_{i}^{(0)} d x^{i}-\left(\bar{\pi}^{(0)} r+\frac{1}{2} \bar{\Psi}_{3}^{(0)} r^{2}\right) \bar{\xi}_{i}^{(0)} d x^{i} \\
& m=-\left(\bar{\pi}^{(0)} r+\frac{1}{2} \Psi_{3}^{(0)} r^{2}\right) d v+\left(1-\mu^{(0)} r\right) \xi_{i}^{(0)} d x^{i}-\left(\bar{\lambda}^{(0)} r+\frac{1}{2} \bar{\Psi}_{4}^{(0)} r^{2}\right) \bar{\xi}_{i}^{(0)} d x^{i}
\end{aligned}
$$

Here $\xi_{i}^{(0)}$ are defined by the relations $\xi_{i}^{(0)} \xi_{(0)}^{i}=0$ and $\xi_{i}^{(0)} \bar{\xi}_{(0)}^{i}=1$; it will be convenient to set $m_{a}^{(0)}:=\xi_{i}^{(0)} \partial_{a} x^{i}$. In powers of $r$, the metric is:

$$
g_{a b}=-2 \ell_{(a} n_{b)}+2 m_{(a} \bar{m}_{b)}=g_{a b}^{(0)}+g_{a b}^{(1)} r+\frac{1}{2} g_{a b}^{(2)} r^{2}+\cdots
$$


The spacetime in the neighborhood of a general isolated black hole

where

$$
\begin{aligned}
g_{a b}^{(0)}= & 2 \partial_{(a} r \partial_{b)} v+2 m_{(a}^{(0)} \bar{m}_{b)}^{(0)} \\
g_{a b}^{(1)}= & -\left(2 \tilde{\kappa} \partial_{(a} v \partial_{b)} v+4 \pi^{(0)} m_{(a}^{(0)} \partial_{b)} v+4 \bar{\pi}^{(0)} \bar{m}_{(a}^{(0)} \partial_{b)} v\right. \\
+ & \left.4 \mu^{(0)} m_{(a}^{(0)} \bar{m}_{b)}^{(0)}+2 \lambda^{(0)} m_{(a}^{(0)} m_{b)}^{(0)}+2 \bar{\lambda}^{(0)} \bar{m}_{(a}^{(0)} \bar{m}_{b)}^{(0)}\right) \\
g_{a b}^{(2)}= & 4\left(\left|\pi^{(0)}\right|^{2}-\operatorname{Re}\left[\Psi_{2}^{(0)}\right]\right) \partial_{(a} v \partial_{b)} v \\
& +4\left(\mu^{(0)} \pi^{(0)}+\lambda^{(0)} \bar{\pi}^{(0)}-\Psi_{3}^{(0)}\right) m_{(a}^{(0)} \partial_{b)} v \\
& +4\left(\mu^{(0)} \bar{\pi}^{(0)}+\bar{\lambda}^{(0)} \pi^{(0)}-\bar{\Psi}_{3}^{(0)}\right) \bar{m}_{(a}^{(0)} \partial_{b)} v \\
& +4\left(\left(\mu^{(0)}\right)^{2}+\left|\lambda^{(0)}\right|^{2}\right) m_{(a}^{(0)} \bar{m}_{b)}^{(0)} \\
& +\left(4 \mu^{(0)} \lambda^{(0)}-2 \Psi_{4}^{(0)}\right) m_{(a}^{(0)} m_{b)}^{(0)}+\left(4 \mu^{(0)} \bar{\lambda}^{(0)}-2 \bar{\Psi}_{4}^{(0)}\right) \bar{m}_{(a}^{(0)} \bar{m}_{b)}^{(0)}
\end{aligned}
$$

We could easily obtain the third order metric by using Eqs. (64), but we shall not do so here.

\section{Inclusion of electromagnetic fields}

Let us now go beyond vacuum spacetimes and include electromagnetic fields. As usual, we expand the Maxwell field components $\phi_{k}$ in powers of $r$ :

$$
\phi_{k}=\phi_{k}^{(0)}+r \phi_{k}^{(1)}+\frac{1}{2} \phi_{k}^{(2)}+\ldots, \quad \text { where } k=0,1,2 .
$$

Consider first the intrinsic horizon geometry. We start with the behavior of the Maxwell field components at the horizon. The Raychaudhuri equation for the null generators, after imposing the conditions of Eqs. (32) and (34) is just one of the Newman-Penrose

field equation (Eq. 43a) that we have used earlier. In the presence of a Maxwell field, that equation becomes

$$
D \rho-\bar{\delta} \kappa=\rho^{2}+|\sigma|^{2}+(\epsilon+\bar{\epsilon}) \rho-2 \alpha \kappa+2\left|\phi_{0}\right|^{2} .
$$

On the horizon, by definition $\rho=\kappa=0$. Thus from the above equation we deduce that we still have $\sigma=0$ as before, and in addition we also get

$$
\phi_{0}^{(0)}=0 \text {. }
$$

This implies that

$$
\Phi_{00}^{(0)}=\Phi_{01}^{(0)}=\Phi_{02}^{(0)}=\Phi_{10}^{(0)}=\Phi_{20}^{(0)}=0 .
$$

Furthermore, we also have $\Lambda=0$ everywhere since the trace of $T_{a b}$ vanishes. Imposing these conditions to determine the time evolution of the geometrical fields on the horizon, the two non-radial Maxwell equations (9a) and (9b) become

$$
\left\{\begin{array}{l}
D \phi_{1}^{(0)}=0 \\
D \phi_{2}^{(0)}+\tilde{\kappa} \phi_{2}^{(0)}=\bar{\delta} \phi_{1}^{(0)}+2 \pi^{(0)} \phi_{1}^{(0)}
\end{array}\right.
$$


Thus, $\phi_{1}^{(0)}$ is time independent, the charges defined in Eq. (23) are constant. Since $\phi_{0}^{(0)}$ vanishes, these charges are in fact independent of which cross-section of $\Delta$ is used in the integral. Just like $\Psi_{3}$ and $\Psi_{4}$ at the horizon, $\phi_{2}$ has a time dependence.

With these simplifications at hand, it turns out that all of Eqs. (47) remain unchanged. The only change in the intrinsic geometry occurs in two of the angular equations (44). The first change is an extra contribution in Eq. (53):

$$
-2 \operatorname{Re}\left[\Psi_{2}^{(0)}\right]+4\left|\phi_{1}^{(0)}\right|^{2}=\delta a+\bar{\delta} \bar{a}-2|a|^{2} \Rightarrow-4 \operatorname{Re}\left[\Psi_{2}^{(0)}\right]+8\left|\phi_{1}^{(0)}\right|^{2}={ }^{2} \tilde{\mathcal{R}} .
$$

The second effect is in Eq. (52) which determines $\Psi_{3}^{(0)}$ :

$$
\Psi_{3}^{(0)}=\overline{\bar{\partial}} \mu^{(0)}+\pi^{(0)} \mu^{(0)}-ð \lambda^{(0)}-\bar{\pi}^{(0)} \lambda^{(0)}+2 \phi_{2}^{(0)} \bar{\phi}_{1}^{(0)} .
$$

The remaining Maxwell equations (9c) and (9d) determine the radial derivatives of $\phi_{0}$ and $\phi_{1}$. As usual, we impose Eqs. (32) and (34) to obtain the radial equations for $\phi_{0}$ and $\phi_{1}$ :

$$
\left\{\begin{array}{l}
\Delta \phi_{0}=\delta \phi_{1}-\mu \phi_{0}+\sigma \phi_{2} \\
\Delta \phi_{1}=\delta \phi_{2}-2 \mu \phi_{1}+2 \beta \phi_{2}
\end{array}\right.
$$

Note that there is no equation for the radial derivative of $\phi_{2}$. Thus, just like $\Psi_{4}$, we need to specify $\psi_{2}$ on the past light cone $\mathcal{N}_{0}$ as part of the free data. As for the other radial equations, we can iterate this to obtain an expansion for $\phi_{0}$ and $\phi_{1}$. To first order we need to simply evaluate the right hand side of these equations at the horizon:

$$
\left\{\begin{array}{l}
\phi_{0}^{(1)}=-\delta \phi_{1}^{(0)} \\
\phi_{1}^{(1)}=-\delta \phi_{2}^{(0)}+2 \mu^{(0)} \phi_{1}^{(0)}+2 \beta^{(0)} \phi_{2}^{(0)}=\left(-ð+\pi^{(0)}\right) \phi_{2}^{(0)}+2 \mu^{(0)} \phi_{1}^{(0)} .
\end{array}\right.
$$

Let us turn now to the Bianchi identities. In the vacuum case, we had the Equations (45) and (46). These equations give here as well the radial and time derivatives of the Weyl tensor components with additional terms for the matter contributions. For the time derivatives at the horizon, we only need to worry about $\Psi_{4}^{(0)}$ (since $\Psi_{3}^{(0)}$ has been determined above and the others are time independent):

$$
\begin{aligned}
D \Psi_{4}^{(0)}+2 \tilde{\kappa} \Psi_{4}^{(0)} & =\left(\overline{\widetilde{\partial}}+5 \pi^{(0)}\right) \Psi_{3}^{(0)}+2 \bar{\phi}_{1}^{(0)}\left(\overline{\widetilde{\partial}}+\pi^{(0)}\right) \phi_{2}^{(0)} \\
& -3 \lambda^{(0)} \Psi_{2}^{(0)}-4 \lambda^{(0)}\left|\phi_{1}^{(0)}\right|^{2} .
\end{aligned}
$$

The radial equations for the Weyl tensor components from the Bianchi identities are not very enlightening, and we shall just write the first derivatives evaluated at the horizon:

$$
\left\{\begin{aligned}
\Psi_{0}^{(1)} & =0 \\
\Psi_{1}^{(1)} & =-\delta \Psi_{2}^{(0)}-2 \bar{\phi}_{1}^{(0)} \delta \phi_{1}^{(0)}, \\
\Psi_{2}^{(1)} & =-\left(\widetilde{\partial}+\bar{\pi}^{(0)}\right) \Psi_{3}^{(0)}-2 \bar{\phi}_{1}^{(0)}\left(ð+\bar{\pi}^{(0)}\right) \phi_{2}^{(0)}+2 \bar{\phi}_{2}^{(0)} \bar{\delta} \phi_{1}^{(0)}+3 \mu \Psi_{2}^{(0)}+4 \mu\left|\phi_{1}^{(0)}\right|^{2}, \\
\Psi_{3}^{(1)} & =-\left(ð+2 \pi^{(0)}\right) \Psi_{4}^{(0)}+4 \mu^{(0)} \Psi_{3}^{(0)}+2 \bar{\phi}_{1}^{(0)} \phi_{2}^{(1)}+2 \bar{\phi}_{2}^{(0)}\left(ð-2 \pi^{(0)}\right) \phi_{2}^{(0)} \\
& -4 \lambda^{(0)} \phi_{1}^{(0)} \bar{\phi}_{2}^{(0)}+4 \pi^{(0)}\left|\phi_{2}^{(0)}\right|^{2}
\end{aligned}\right.
$$


In the presence of matter fields, there are three (two real and one complex) additional Bianchi identities containing only Ricci tensor components. Let us consider these equations at the horizon after imposing our conditions on the spin coefficients and the vanishing of $\phi_{0}$. The first of these equations yields simply that $\phi_{0}^{(0)}$ is time independent (which we already knew):

$$
D \Phi_{11}^{(0)}=0 \Rightarrow D \phi_{1}^{(0)}=0 .
$$

The second determines the time evolution of $\Phi_{12}^{(0)}$

$$
D \Phi_{12}^{(0)}-\delta \Phi_{11}^{(0)}+\Delta \Phi_{01}^{(0)}=2 \bar{\pi}^{(0)} \Phi_{11}^{(0)}-2 \bar{\epsilon}^{(0)} \Phi_{12}^{(0)} .
$$

The third yields the time dependence of $\Phi_{22}$ :

$$
\begin{aligned}
D \Phi_{22}^{(0)}-\delta \Phi_{21}^{(0)}+\Delta \Phi_{11}^{(0)}-\bar{\delta} \Phi_{12}^{(0)} & =-4 \mu^{(0)} \Phi_{11}^{(0)}+\left(2 \pi^{(0)}+2 \bar{\beta}^{(0)}\right) \Phi_{12}^{(0)} \\
& +\left(2 \bar{\pi}^{(0)}+2 \beta^{(0)}\right) \Phi_{21}^{(0)}-4 \epsilon^{(0)} \Phi_{22}^{(0)}
\end{aligned}
$$

Using $\Phi_{i j}=2 \phi_{i} \bar{\phi}_{j}$, it can be shown that these two equations do not have any new information and can be obtained by combining Eqs. (85) and (88).

In the rest of this section, let us us focus on the expansion of the metric up to $\mathcal{O}\left(r^{2}\right)$. First we need the radial derivatives of the spin coefficients as in Eqs. (63). Let us rewrite Eqs. (63) keeping this time the contributions of $\phi_{1}$ and $\phi_{2}$ at the horizon, and focusing on the spin coefficients which is all we need for expanding the metric up to second order:

$$
\left\{\begin{array}{l}
\kappa^{(1)}=\sigma^{(1)}=0 \\
\rho^{(1)}=\Psi_{2}^{(0)} \\
\epsilon^{(1)}+\bar{\epsilon}^{(1)}=2\left|\pi^{(0)}\right|^{2}+2 \operatorname{Re}\left[\Psi_{2}^{(0)}\right]+4\left|\phi_{1}^{(0)}\right|^{2} \\
\epsilon^{(1)}-\bar{\epsilon}^{(1)}=\bar{\pi}^{(0)}\left(\alpha^{(0)}-\bar{\beta}^{(0)}\right)-\pi^{(0)}\left(\bar{\alpha}^{(0)}-\beta^{(0)}\right)+\Psi_{2}^{(0)}-\bar{\Psi}_{2}^{(0)} \\
\lambda^{(1)}=2 \mu^{(0)} \lambda^{(0)}+\Psi_{4}^{(0)} \\
\mu^{(1)}=\left(\mu^{(0)}\right)^{2}+\left|\lambda^{(0)}\right|^{2}+2 \|\left.\phi_{2}^{(0)}\right|^{2} \\
\pi^{(1)}=\alpha^{(1)}+\bar{\beta}^{(1)}=\pi^{(0)} \mu^{(0)}+\bar{\pi}^{(0)} \lambda^{(0)}+\Psi_{3}^{(0)}+2 \phi_{2}^{(0)} \bar{\phi}_{1}^{(0)} \\
\alpha^{(1)}-\bar{\beta}^{(1)}=\mu^{(0)}\left(\alpha^{(0)}-\bar{\beta}^{(0)}\right)+\lambda^{(0)}\left(\beta^{(0)}-\bar{\alpha}^{(0)}\right)+\Psi_{3}^{(0)}+2 \phi_{2}^{(0)} \bar{\phi}_{1}^{(0)}
\end{array}\right.
$$

We now need the radial derivatives for the frame functions given in Eqs. (40) which leads us to the expansion of the frame functions:

$$
\left\{\begin{aligned}
U= & r \tilde{\kappa}+r^{2}\left(2\left|\pi^{(0)}\right|^{2}+\operatorname{Re}\left[\Psi_{2}^{(0)}\right]+2\left|\phi_{1}^{(0)}\right|^{2}\right)+\mathcal{O}\left(r^{3}\right) \\
\Omega= & r \bar{\pi}^{(0)}+r^{2}\left(\mu^{(0)} \bar{\pi}^{(0)}+\bar{\lambda}^{(0)} \pi^{(0)}+\frac{1}{2} \bar{\Psi}_{3}^{(0)}+\phi_{1}^{(0)} \bar{\phi}_{2}^{(0)}\right)+\mathcal{O}\left(r^{3}\right) \\
X^{i}= & \bar{\Omega} \xi_{(0)}^{i}+\Omega \bar{\xi}_{(0)}^{i}+\mathcal{O}\left(r^{3}\right) \\
\xi^{i}= & {\left[1+r \mu^{(0)}+r^{2}\left(\left(\mu^{(0)}\right)^{2}+\left|\lambda^{(0)}\right|^{2}+\left|\phi_{2}^{(0)}\right|^{2}\right)\right] \xi_{(0)}^{i} } \\
& +\left[r \bar{\lambda}^{(0)}+r^{2}\left(2 \mu^{(0)} \bar{\lambda}^{(0)}+\frac{1}{2} \bar{\Psi}_{4}^{(0)}\right)\right] \bar{\xi}_{(0)}^{i}+\mathcal{O}\left(r^{3}\right)
\end{aligned}\right.
$$


It is instructive to compare this with Eqs. (69). We see that the electromagnetic field modifies $\Psi_{2}$ and $\Psi_{3}$ according to

$$
\operatorname{Re}\left[\Psi_{2}^{(0)}\right] \rightarrow \operatorname{Re}\left[\Psi_{2}^{(0)}\right]+2\left|\phi_{1}^{(0)}\right|^{2}, \quad \Psi_{3}^{(0)} \rightarrow \Psi_{3}^{(0)}+2 \phi_{1}^{(0)} \bar{\phi}_{2}^{(0)}
$$

In addition, the $\left(\mu^{(0)}\right)^{2}+\left|\lambda^{(0)}\right|^{2}$ term gets an additive correction by $\left|\phi_{2}^{(0)}\right|^{2}$.

We can finally write the expansion of the metric. It is clear that there is no change up to $\mathcal{O}(r)$. Just like the Weyl tensor, the effects of the electromagnetic field first enter the metric at second order. Thus, Eqs. (78) and (78) are unchanged and Eq. (80) becomes:

$$
\begin{aligned}
g_{a b}^{(2)} & =4\left(\left|\pi^{(0)}\right|^{2}-\operatorname{Re}\left[\Psi_{2}^{(0)}\right]+2\left|\phi_{1}^{(0)}\right|^{2}\right) \partial_{(a} v \partial_{b)} v \\
& +4\left(\mu^{(0)} \pi^{(0)}+\lambda^{(0)} \bar{\pi}^{(0)}-\Psi_{3}^{(0)}-2 \phi_{2}^{(0)} \bar{\phi}_{1}^{(0)}\right) m_{(a}^{(0)} \partial_{b)} v \\
& +4\left(\mu^{(0)} \bar{\pi}^{(0)}+\bar{\lambda}^{(0)} \pi^{(0)}-\bar{\Psi}_{3}^{(0)}-2 \bar{\phi}_{2}^{(0)} \phi_{1}^{(0)}\right) \bar{m}_{(a}^{(0)} \partial_{b)} v \\
& +4\left(\left(\mu^{(0)}\right)^{2}+\left|\lambda^{(0)}\right|^{2}+\left|\phi_{2}^{(0)}\right|^{2}\right) m_{(a}^{(0)} \bar{m}_{b)}^{(0)} \\
& +\left(4 \mu^{(0)} \lambda^{(0)}-2 \Psi_{4}^{(0)}\right) m_{(a}^{(0)} m_{b)}^{(0)}+\left(4 \mu^{(0)} \bar{\lambda}^{(0)}-2 \bar{\Psi}_{4}^{(0)}\right) \bar{m}_{(a}^{(0)} \bar{m}_{b)}^{(0)}
\end{aligned}
$$

\section{Conclusions}

In this paper we have investigated the geometry in the vicinity of a non-extremal weakly isolated horizon using the Newman-Penrose formalism. We allow the horizon to have generic values of mass, spin and charge and higher multipole moments. We have obtained the connection, metric and the Weyl tensor up to $\mathcal{O}\left(r^{2}\right)$. On the horizon, we choose a parametrization of the null generators so that we get a specific value of the surface gravity. In addition, on an initial spherical cross section $S_{0}$ of the horizon, we specify

the spin coefficients $\pi, \alpha-\bar{\beta}, \mu, \lambda$. The spin coefficients $\pi$ and $\alpha-\bar{\beta}$ out to be time independent and they specify respectively the $\operatorname{Im}\left[\Psi_{2}\right]$ and the scalar curvature of $S_{0}$, and thus the spin and mass multipole moments of the horizon respectively. The transversal expansion and shear $\mu, \lambda$ turn out to be time dependent and they determine $\Psi_{3}$. In addition, we need to specify $\Psi_{4}$ on the past-outgoing light cone $\mathcal{N}_{0}$ originating from $S_{0}$. If we consider charged horizons, then we must specify in addition the Maxwell field component $\phi_{1}$ on $S_{0}$ (this determines the electric and magnetic charges of the horizon) and $\phi_{2}$ on $\mathcal{N}_{0}$.

We emphasize once again that this is a local calculation and not all solutions produced by this construction are physically meaningful; the interesting issue of global existence of solutions has not been addressed here. For example, the uniqueness theorems would imply that not all values of the horizon multipole moments would lead to asymptotically flat solutions. However, the set of solution we have obtained encompasses all physically interesting situations (in so far as the assumption of the horizon being isolated is valid).

There are a number of possible extensions of this work. The first is the study of tidally deformed black holes which has, among other things, implications for the the equation of motion and self force in general relativity [35]. This requires us to consider 
Kerr black holes perturbed by a background curvature, and to interpret the resulting metric in terms of a moving point particle. This will be studied in a forthcoming paper. The near horizon geometry computed here could be matched to an appropriate far zone metric to obtain initial data for numerical simulations of binary black hole systems (see e.g. 36]) and finally, future gravitational wave observatories might be able to measure the tidal deformations through observations of signals from extreme mass ratio systems. Knowledge of the near horizon geometry including deviations from the Kerr multipoles values (which are allowed here) will be useful in the searches for these signals and in testing the Kerr nature of the black hole. Finally beyond the specific calculation of the near horizon metric, the characteristic initial value formulation based on the horizon might be useful for wave extraction in numerical relativity signals. If $S_{0}$ is taken to be a cross-section of the horizon at very late times, then the light cone $\mathcal{N}_{0}$ can be viewed as an approximate null infinity. The usefulness of this construction remains to be explored.

\section{Acknowledgments}

I thank Abhay Ashtekar for valuable suggestions and discussions. I am also grateful to Bernard Schutz, Toshifumi Futamase, Dirk Pützfeld and Stas Babak for discussions regarding possible applications of this work.

\section{References}

[1] Ashtekar A, Beetle C, Fairhurst S. Isolated horizons: A generalization of black hole mechanics. Class Quant Grav. 1999;16:L1-L7.

[2] Ashtekar A, Beetle C, Fairhurst S. Mechanics of Isolated Horizons. Class Quant Grav. 2000;17:253-298.

[3] Ashtekar A, Fairhurst S, Krishnan B. Isolated horizons: Hamiltonian evolution and the first law. Phys Rev. 2000;D62:104025.

[4] Ashtekar A, et al. Isolated horizons and their applications. Phys Rev Lett. 2000;85:3564-3567.

[5] Ashtekar A, Beetle C, Lewandowski J. Mechanics of Rotating Isolated Horizons. Phys Rev. 2001;D64:044016.

[6] Ashtekar A, Beetle C, Lewandowski J. Geometry of Generic Isolated Horizons. Class Quant Grav. 2002;19:1195-1225.

[7] Adamo TM, Newman ET. Vacuum non-expanding horizons and shear-free null geodesic congruences. Class Quant Grav. 2009;26:235012.

[8] Hayward SA. General laws of black hole dynamics. PhysRev. 1994;D49:6467-6474.

[9] Ashtekar A, Krishnan B. Dynamical horizons: Energy, angular momentum, fluxes and balance laws. Phys Rev Lett. 2002;89:261101.

[10] Ashtekar A, Krishnan B. Isolated and dynamical horizons and their applications. Living Rev Rel. 2004;7:10.

[11] Booth I. Black hole boundaries. Can J Phys. 2005;83:1073-1099.

[12] Gourgoulhon E, Jaramillo JL. A 3+1 perspective on null hypersurfaces and isolated horizons. Phys Rept. 2006;423:159-294.

[13] Hayward SA. Dynamics of black holes; 2008.

[14] Comeau S, Poisson E. Tidal interaction of a small black hole in the field of a large Kerr black hole. PhysRev. 2009;D80:087501. 
[15] Poisson E, Vlasov I. Geometry and dynamics of a tidally deformed black hole. Phys Rev. 2010;D81:024029.

[16] Poisson E. Metric of a tidally distorted, nonrotating black hole. PhysRevLett. 2005;94:161103.

[17] Yunes N, Gonzalez JA. Metric of a tidally perturbed spinning black hole. PhysRev. 2006;D73:024010.

[18] Damour T, Lecian OM. On the gravitational polarizability of black holes. PhysRev. 2009;D80:044017.

[19] Bondi H, van der Burg MGJ, Metzner AWK. Gravitational waves in general relativity. 7. Waves from axisymmetric isolated systems. Proc Roy Soc Lond. 1962;A269:21-52.

[20] Friedrich H. On the Regular and the Asymptotic Characteristic Initial Value Problem for Einstein's Vacuum Field Equations. Proc Roy Soc Lond. 1981;A375:169-184.

[21] Rendall AD. Reduction of the characteristic initial value problem to the Cauchy problem and its applications to the Einstein equations. Proc Roy Soc Lond. 1990;427:221-239.

[22] Friedrich H, Rendall AD. The Cauchy problem for the Einstein equations. LectNotes Phys. 2000;540:127-224. Einstein's Field Equations and their Physical Interpretation, ed. by B. G. Schmidt, Springer, Berlin, 2000.

[23] Stewart J. Advanced General Relativity. Cambridge Monographs on Mathematical Physics; 1991.

[24] Lewandowski J. Spacetimes Admitting Isolated Horizons. Class Quant Grav. 2000;17:L53-L59.

[25] Newman E, Penrose R. An Approach to gravitational radiation by a method of spin coefficients. J Math Phys. 1962;3:566-578.

[26] Diener P;. Private communication.

[27] Newman ET, Unti TWJ. Behavior of asymptotically flat empty space. J Math Phys. 1962;3:891901.

[28] Penrose R, Rindler W. Spinors and Stacetime: 1. Two-Spinor Calculus and Relativistic Fields. Cambridge, Uk: Univ. Pr. ( 1984) 458 P. ( Cambridge Monographs On Mathematical Physics); 1984.

[29] Chandrasekhar S. The mathematical theory of black holes. Oxford Classic Texts in the Physical Sciences; 1985.

[30] Goldberg JN, MacFarlane AJ, Newman ET, Rohrlich F, Sudarshan ECG. Spin s spherical harmonics and edth. J Math Phys. 1967;8:2155.

[31] Dreyer O, Krishnan B, Shoemaker D, Schnetter E. Introduction to Isolated Horizons in Numerical Relativity. Phys Rev. 2003;D67:024018.

[32] Beetle C. Approximate Killing Fields as an Eigenvalue Problem; 2008.

[33] Cook GB, Whiting BF. Approximate Killing Vectors on S**2. PhysRev. 2007;D76:041501.

[34] Ashtekar A, Engle J, Pawlowski T, Van Den Broeck C. Multipole moments of isolated horizons. Class Quant Grav. 2004;21:2549-2570.

[35] Poisson E. The motion of point particles in curved spacetime. Living Rev Rel. 2004;7:6.

[36] Yunes N, Tichy W. Improved initial data for black hole binaries by asymptotic matching of post-Newtonian and perturbed black hole solutions. PhysRev. 2006;D74:064013. 